\title{
Horizontal transfer and proliferation of Tsu4 in Saccharomyces paradoxus
}

\author{
Casey M. Bergman (1)
}

\begin{abstract}
Background: Recent evidence suggests that horizontal transfer plays a significant role in the evolution of of transposable elements (TEs) in eukaryotes. Many cases of horizontal TE transfer (HTT) been reported in animals and plants, however surprisingly few examples of $\mathrm{HTT}$ have been reported in fungi.

Findings: Here I report evidence for a novel HTT event in fungi involving Tsu4 in Saccharomyces paradoxus based on (i) unexpectedly high similarity between Tsu4 elements in S. paradoxus and S. uvarum, (ii) a patchy distribution of Tsu4 in S. paradoxus and general absence from its sister species S. cerevisiae, and (iii) discordance between the phylogenetic history of Tsu4 sequences and species in the Saccharomyces sensu stricto group. Available data suggests the HTT event likely occurred somewhere in the Nearctic, Neotropic or Indo-Australian part of the S. paradoxus species range, and that a lineage related to S. uvarum or S. eubayanus was the likely donor species. The HTT event has led to massive proliferation of Tsu4 in the South American lineage of S. paradoxus, which exhibits partial reproductive isolation with other strains of this species because of multiple reciprocal translocations. Full-length Tsu4 elements are associated with both breakpoints of one of these reciprocal translocations.

Conclusions: This work shows that comprehensive analysis of TE sequences in essentially-complete genome assemblies derived from long-read sequencing provides new opportunities to detect HTT events in fungi and other organisms. This work also provides support for the hypothesis that HTT and subsequent TE proliferation can induce genome rearrangements that contribute to post-zygotic isolation in yeast.
\end{abstract}

Keywords: Transposable element, Horizontal transfer, Yeast, Genome rearrangement

\section{Main Text}

Horizontal transfer is increasingly thought to play an important role in shaping the diversity of transposable elements (TEs) in eukaryotic genomes [1-3]. Since the initial discovery of horizontal transfer of the $P$ element from Drosophila willistoni to D. melanogaster [4], a large number of cases of horizontal TE transfer (HTT) have been reported, especially among animals species (data compiled in [5]). However, surprisingly few cases of HTT have been reported in fungi [6-12], despite an abundance of genomic resources in this taxonomic group. Advances in long-read whole genome shotgun sequencing now allow comprehensive analysis of TE sequences in high-quality genome assemblies, and may therefore

\footnotetext{
Correspondence: cbergman@uga.edu

Department of Genetics and Institute of Bioinformatics, University of Georgia, East Green St., 30602 Athens, GA, USA
}

provide new opportunities for detecting HTT events in fungi and other organisms.

For example, a recent study by Yue et al. [13] reported essentially-complete PacBio genome assemblies for seven strains of $S$. cerevisiae and five strains of S. paradoxus. Analysis of TEs in these assemblies revealed a surprisingly high copy number for the $T y 4$ family in one strain of S. paradoxus from South America (UFRJ50816; $n=23$ copies) [13]. This was a noteworthy observation for two reasons: (i) Ty4 is typically found at low copy number in yeast strains [14-16], and (ii) S. American strains of S. paradoxus exhibit partial reproductive isolation with other strains of this species, which principally results from multiple reciprocal translocations thought to have arisen by unequal crossing-over between dispersed repetitive elements such as Ty elements [17].

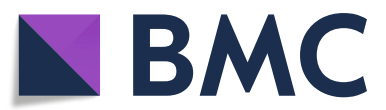

(c) The Author(s). 2018 Open Access This article is distributed under the terms of the Creative Commons Attribution 4.0 International License (http://creativecommons.org/licenses/by/4.0/), which permits unrestricted use, distribution, and reproduction in any medium, provided you give appropriate credit to the original author(s) and the source, provide a link to the Creative Commons license, and indicate if changes were made. The Creative Commons Public Domain Dedication waiver (http://creativecommons.org/publicdomain/zero/1.0/) applies to the data made available in this article, unless otherwise stated. 
I independently replicated the curious observation of exceptionally high Ty4 copy number in S. paradoxus UFRJ50816 using a RepeatMasker-based annotation pipeline similar to that described in [11], which identifies and classifies $T y$ elements as full-length, truncated, or solo long terminal repeats (LTRs). Using the results of this initial annotation, I generated a multiple alignment of all full-length Ty4 elements identified in these 12 assemblies. Preliminary phylogenetic analysis revealed that the full-length Ty4 elements from S. paradoxus UFRJ50816 formed a monophyletic clade of very similar sequences that were highly divergent from other full-length Ty4 elements identified in S. cerevisiae (S288c, Y12, YPS128) or S. paradoxus (N44). Surprisingly, BLAST analysis at NCBI using representative members of this divergent Ty4-like clade revealed that they were more similar to the Tsu4 element from the related yeast species $S$. uvarum (Genbank: AJ439550) [18] than they were to the original S. cerevisiae Ty4 query sequence (Genbank: S50671). This result suggested that the unusually high copy number of $T y 4$ in $S$. paradoxus UFRJ50816 reported by Yue et al. [13] could actually be the consequence of rapid expansion of Tsu4 following a HTT event from a S. uvarum-like donor.

To better characterize Ty4 and Tsu4 content in S. cerevisiae and S. paradoxus, I first identified a canonical S. paradoxus Tsu4 element. To do this, I included the S. uvarum Tsu4 query sequence in the TE library from [11] and re-annotated Ty elements in S. paradoxus UFRJ50816 using the same RepeatMasker-based strategy as above. I also performed de novo identification of full-length LTR elements in S. paradoxus UFRJ50816 using LTRharvest [19], then overlapped results from RepeatMasker and LTRharvest to identify full-length Tsu4 elements, generated a consensus sequence from these elements, and finally identified the genomic copy (chrII:554570-560566) that clustered most closely with the consensus sequence of full-length Tsu4 elements in a neighbor-joining tree. The canonical S. paradoxus Tsu4 element is 5,997 bp in length, with $293 \mathrm{bp}$ LTRs and two overlapping reading frames predicted to encode GAG (358 amino acids) and POL (1419 amino acids). Codon-based alignment with PRANK [20] and estimation of $\mathrm{dN} / \mathrm{dS}$ ratios under PAML model M0 using ETE $[21,22]$ revealed that selective constraints have operated on both GAG (0.34) and POL (0.21) sequence divergence between $S$. uvarum and S. paradoxus Tsu4 canonical elements. Estimates of dS for GAG (0.23) and POL (0.24) between S. uvarum and S. paradoxus Tsu4 canonical elements were over four times lower than the median dS (1.01) for nuclear coding sequences estimated under M0 for these species using 4554 high-confidence alignments from [23]. This observation supports HTT of Tsu4 and argues against various modes of vertical inheritance to explain the similarity between $S$. paradoxus and S. uvarum Tsu4.
I then performed a final annotation of $T y$ elements in all 12 assemblies from Yue et al. [13] using the TE library from [11] plus the newly-identified S. paradoxus Tsu4 canonical element. Full-length, truncated and solo LTR counts for Tsu4 and Ty4 can be found in Table 1. Similar data for all $T y$ families in these genomes can be found in Additional file 1 and coordinates of all annotated Ty elements in these genomes can be found in Additional file 2. This improved annotation revealed a low copy number of full-length Ty4 elements for S. cerevisiae S288c, Y12, and YPS128 as well as S. paradoxus N44, which is typical of this family [14-16]. Solo LTRs for Ty4 were found in all strains of S. cerevisiae and S. paradoxus with PacBio data from from Yue et al. [13]. Solo LTRs arise by intra-element LTR-LTR recombination and serve as useful markers of past transpositional activity [24]. These results suggest that $T y 4$ was present in the common ancestor of both $S$. cerevisiae and S. paradoxus and that this family has been maintained at low copy number or become inactive in different lineages of each species.

In contrast, I found evidence for full-length Tsu4 sequences in only three strains of $S$. paradoxus from $S$. America (UFRJ50816, $n=22$ ), N. America (YPS138, $n=1$ ) and Hawaii (UWOPS91-917.1, $n=1$ ) (Table 1). S. American, N. American, and Hawaiian lineages of $S$. paradoxus form a monophyletic group that is distinct from a clade containing European and Far-Eastern lineages from the Old World [13, 25]. S. paradoxus strains with full-length copies of Tsu4 were devoid of full-length Ty4 elements, and vice versa. Crucially, only these three S. paradoxus strains had solo LTRs for Tsu4, suggesting they are the only lineages in which Tsu4 has been active in the past. Consistent with a more recent presence in the $S$. paradoxus genome, solo LTRs for Tsu4 exhibited $\geq 3$ fold lower average pairwise divergence than Ty4 solo LTRs relative to their respective canonical elements within the genomes of UFRJ50816 (Tsu4: 0.02, Ty4: 0.10), YPS138 (Tsu4: 0.03, Ty4: 0.10) and UWOPS91-917.1 (Tsu4: 0.04, Ty4: 0.12).

The 22 full-length copies of Tsu4 identified in UFRJ50816 are distributed on 11 different chromosomes, indicating proliferation in UFRJ50816 is not simply due to tandem duplication. Twelve of the full-length Tsu4 copies in UFRJ50816 are flanked by 5 bp target site duplications (TSDs) that can be automatically identified by LTRharvest [19] and 21 are within $\pm 1 \mathrm{~kb}$ of a tRNA gene, similar to what has been reported previously for $\mathrm{Ty} 4 \mathrm{in}$ S. cerevisiae $[15,26]$. Diagnostic hallmarks of active Ty element transposition such as 5 bp TSDs and tRNA targeting suggest that Tsu4 has been recently active in UFRJ50816 and also argue against the possibility that $T s u 4$ sequences in $S$. paradoxus are due to contamination in DNA samples.

I next confirmed the general absence of Tsu4 in S. cerevisiae by BLAST analysis of an additional 336 S. cerevisiae whole 
Table 1 Ty4 and Tsu4 content in S. cerevisiae and S. paradoxus PacBio assemblies from Yue et al. [13]

\begin{tabular}{|c|c|c|c|c|c|c|c|}
\hline Species & Strain & \# Ty4 full & \# Ty4 truncated & \# Ty4 solo LTR & \# Tsu4 full & \# Tsu4 truncated & \# Tsu4 solo LTR \\
\hline Scer & S288c & 3 & 0 & 14 & 0 & 0 & 0 \\
\hline Scer & DBVPG6044 & 0 & 0 & 18 & 0 & 0 & 0 \\
\hline Scer & DBVPG6765 & 0 & 0 & 5 & 0 & 0 & 0 \\
\hline Scer & SK1 & 0 & 0 & 14 & 0 & 0 & 0 \\
\hline Scer & Y12 & 4 & 1 & 14 & 0 & 0 & 0 \\
\hline Scer & YPS128 & 4 & 0 & 13 & 0 & 0 & 0 \\
\hline Scer & UWOPS03-461.4 & 0 & 0 & 60 & 0 & 0 & 0 \\
\hline Spar & CBS432 & 0 & 2 & 46 & 0 & 0 & 0 \\
\hline Spar & N44 & 3 & 3 & 106 & 0 & 0 & 0 \\
\hline Spar & YPS138 & 0 & 0 & 49 & 1 & 0 & 18 \\
\hline Spar & UFRJ50816 & 0 & 1 & 45 & 22 & 2 & 105 \\
\hline Spar & UWOPS91-917.1 & 0 & 0 & 40 & 1 & 1 & 88 \\
\hline
\end{tabular}

Numbers of full-length elements (both LTRs present and internal region present with > 95\% coverage of canonical internal region), truncated elements (internal region present with < 95\% coverage of canonical internal region), or solo LTRs (no match to internal region) were estimated using a RepeatMasker (version 4.0.5) based strategy and a custom library of Ty elements from [11] supplemented with S. paradoxus Tsu4

genome shotgun (WGS) assemblies at NCBI (taxid: 4932), which revealed only one nearly complete sequence with high similarity to Tsu4 from a S. cerevisiae strain isolated from a rum distillery in the West Indies ( $>80 \%$ coverage and $>80 \%$ identity, see below) [27]. The patchy distribution of Tsu4 sequences in S. paradoxus and general absence from $S$. cerevisiae suggests that this element was not present in the common ancestor of these species, and instead was recently acquired by a $S$. paradoxus lineage somewhere in the Nearctic, Neotropic or Indo-Australian region, possibly in a strain lacking an active Ty4. In principle, the patchy distribution of Tsu4 in S. paradoxus and absence from $S$. cerevisiae could be explained by vertical inheritance from the common ancestor of $S$. uvarum, S. cerevisiae and S. paradoxus followed by multiple losses in S. cerevisiae and S. paradoxus, however the much lower dS between S. uvarum and S. paradoxus Tsu4 coding regions relative to host genes argues against this scenario.

To provide further support for the hypothesis that Tsu4 recently invaded $S$. paradoxus by HTT, I constructed a maximum likelihood phylogeny of all full-length Ty4 and Tsu4 sequences identified in the 12 strains of S. cerevisiae and S. paradoxus from Yue et al. [13] using RAxML [28]. In this analysis, I also included all complete or nearlycomplete Tsu4 elements identified by BLAST in 392 Saccharomyces WGS assemblies at NCBI (taxid: 4930) that had high similarity to the S. uvarum Tsu4 query sequence $(>80 \%$ coverage and $>80 \%$ identity). These additional 12 Tsu4 sequences include three sequences from the same strain of $S$. uvarum in which Tsu4 was discovered, one sequence from S. mikatae, one sequence from S. kudriavzevii, one sequence each from four strains of $S$. pastorianus, two sequences from an unknown Saccharomyces species (strain M14) isolated in China that is involved in lager brewing, and the single sequence from $S$. cerevisiae (strain 245) mentioned above (Additional file 3) [27, 29-31]. S. mikatae is the most closely related outgroup species to the $S$. cerevisiae/S. paradoxus clade, followed by $S$. kudriavzevii, then a clade containing $S$. uvarum and $S$. eubayanus (reviewed in [32]). S. pastorianus is a hybrid species used in lager brewing containing subgenomes from S. cerevisiae and S. eubayanus $[30,33,34]$. The multiple sequence alignment and maximum likelihood tree for this dataset can be found in Additional files 4 and 5 , respectively.

Figure 1a clearly shows that Tsu4-like sequences form a well-supported monophyletic clade that is distinct from the Ty4 lineage present in S. cerevisiae and S. paradoxus. All S. paradoxus Tsu4 sequences form a single clade that also contains the Tsu4 sequence from S. cerevisiae strain 245, suggesting one initial HTT event into S. paradoxus followed by a secondary HTT event from $S$. paradoxus into $S$. cerevisiae. The most closely-related lineage to the S. paradoxus Tsu4 clade is a clade containing sequences from S. pastorianus and Saccharomyces sp. M14, followed by a clade containing sequences from $S$. uvarum. The grouping of Tsu4 sequences from the hybrid species $S$. pastorianus with those from $S$. paradoxus and S. uvarum can most parsimoniously be explained if Tsu4 sequences from $S$. pastorianus are derived from the $S$. eubayanus component of the hybrid genome (see below). If this is true, then Tsu4 in S. paradoxus could plausibly have arisen via HTT from S. eubayanus, the sister species to $S$. uvarum. Tsu4 sequences from both $S$. mikatae and S. kudriavzevii are outgroups to the crown Tsu4 lineage, but group more closely to the Tsu4 


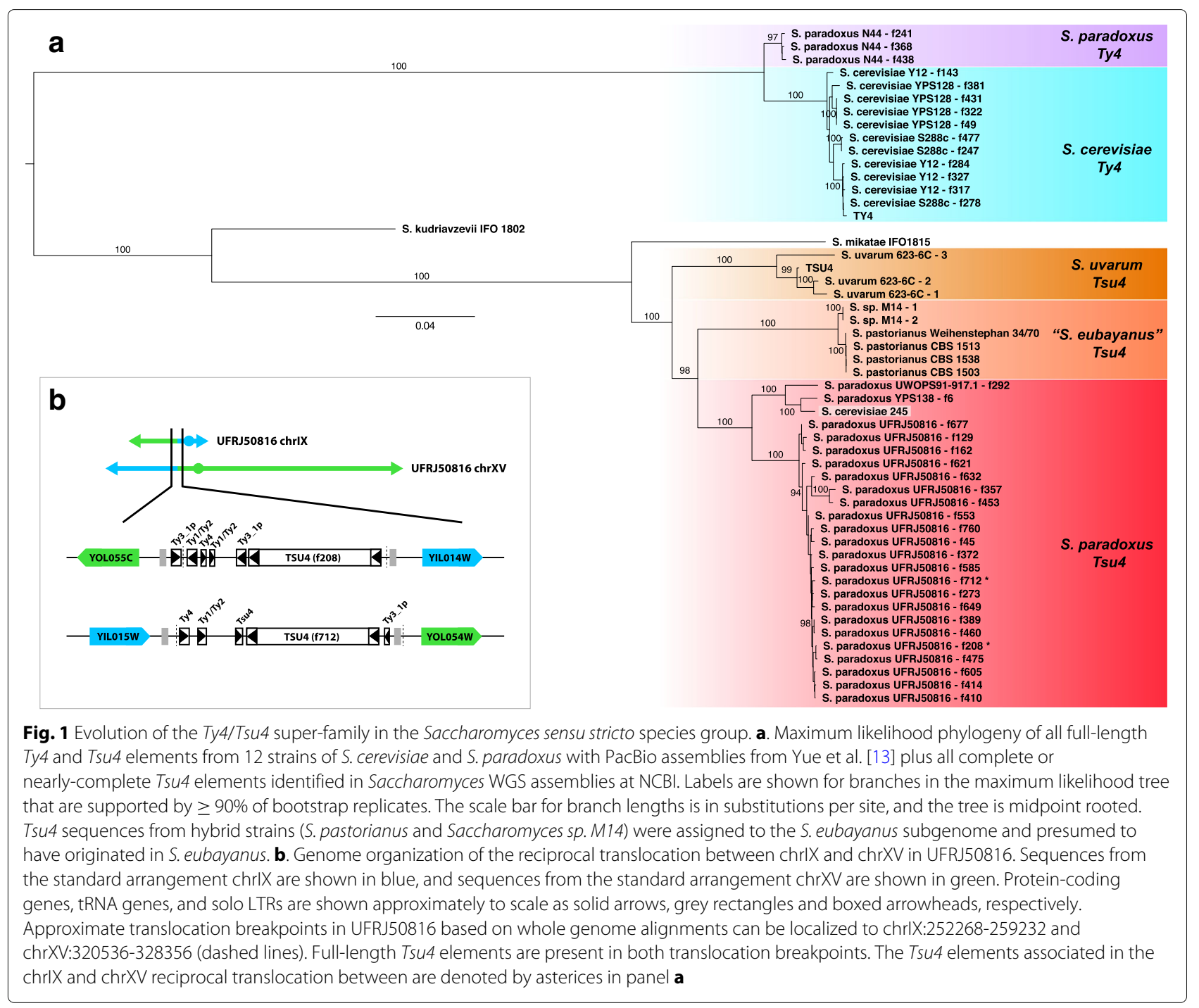

lineage than to the Ty4 lineage with strong support. The observation that $S$. mikatae groups more closely Tsu4 with the crown Tsu4 lineage than S. kudriavzevii is incompatible with the accepted species tree [32], suggesting unequal rates of evolution or another potential HTT event involving Tsu4 between $S$. mikatae and the ancestor of $S$. uvarum and S. eubayanus. Despite unresolved issues with some aspects of the current Tsu4 phylogeny, the fact that $S$. uvarum is the closest pure species clustering with the $S$. paradoxus clade is clearly incompatible with the accepted tree for these species [32] and this discordance provides support for the conclusion that Tsu4 arose in S. paradoxus by HTT from $S$. uvarum or a closely related species like $S$. eubayanus.

To address the origin of Tsu4 sequences in S. pastorianus genomes and better understand potential donors for the HTT event, I aligned S. pastorianus WGS assemblies to a pan-genome comprised of S. cerevisiae S288c [13] and S. eubayanus FM1318 [34], a monosporic derivative of the S. eubayanus type strain which was isolated from Northwestern Patagonia, Argentina [33]. The Tsu4 sequences from all four strains of S. pastorianus are contained on scaffolds that align best to scaffolds from the $S$. eubayanus subgenome (Additional file 6), consistent with the general lack of Tsu4 in S. cerevisiae and the phylogenetic clustering of S. pastorianus Tsu4 sequences with those from S. uvarum. In fact, Tsu4 sequences for all four $S$. pastorianus strains align to the same location in $S$. eubayanus (scaffold NC_030972.1), which together with their tight clustering on the tree suggests they are alleles of the same insertion event. Likewise, alignment of the Saccharomyces sp. M14 genome to a pan-genome of $S$. eubayanus and S. cerevisiae revealed large scaffolds that aligned to either scaffolds from S. eubayanus or chromosomes from S. cerevisiae (Additional file 7), in a similar pattern to the bona fide S. pastorianus group 2/Frohberg 
strain W34/70 (Additional file 8). Thus, Saccharomyces sp. M14 appears to be a hybrid of $S$. cerevisiae and $S$. eubayanus and may possibly be a previously-unidentified strain of S. pastorianus. The two Tsu4 sequences from Saccharomyces sp. M14 align to a different S. eubayanus scaffold (NC_030977.1) and form a cluster on the tree that is distinct from the $S$. pastorianus Tsu4 sequences, suggesting they arose from different transposition events. None of the S. pastorianus/Saccharomyces sp. M14 Tsu4 insertions are found in the $S$. eubayanus reference genome, nor are any other complete or nearly-complete Tsu4 sequences. Several distinct clades have been identified in S. eubayanus using whole genome sequence data: the reference strain (FM1318) is found in the Patagonia B-1 clade, while North American, Tibetan and the $S$. eubayanus subgenomes of $S$. pastorianus strains are found in the related but distinct Holoarctic clade [35]. Overall, the $S$. eubayanus subgenome localization and close affinity of S. pastorianus/Saccharomyces sp. M14 Tsu4 sequences with $S$. paradoxus Tsu4 sequences suggest that S. eubayanus is a viable donor for the Tsu4 element that invaded S. paradoxus, and that Tsu4 has been recently active in the Holoarctic clade of S. eubayanus.

Within S. paradoxus, the deepest well-supported branches in the S. paradoxus Tsu4 clade are between N. American/Hawaiian and S. American S. paradoxus lineages, suggesting the HTT event predates separation of these lineages and could therefore have occurred anywhere in the ancestral range for S. paradoxus in the Nearctic, Neotropic or Indo-Australian regions. All of the Tsu4 sequences in UFRJ50816 form a single clade, but bootstrap support for most branches within this clade are low, consistent with a recent proliferation event occurring after separation of S. American S. paradoxus from N. American and Hawaiian lineages. The single S. cerevisiae Tsu4 sequence found in a strain 245 (isolated from the French West Indies) clusters strongly with the Tsu4 sequence from the N. American S. paradoxus strain YPS138. Introgression of S. paradoxus DNA into S. cerevisiae has been observed previously [36-41], and thus introgression from a N. American-like lineage of S. paradoxus Tsu4 into $S$. cerevisiae in the Caribbean could explain this secondary HTT event. As in N. American and Hawaiian lineages of S. paradoxus, Tsu4 in this S. cerevisiae lineage has not led to widespread proliferation, suggesting the high copy number of Tsu4 in UFRJ50816 is exceptional.

S. paradoxus UFRJ50816 was originally thought to represent a distinct species called S. cariocanus based on partial reproductive isolation with $S$. paradoxus tester strains $[36,42,43]$. Five reciprocal translocations have been identified on the lineage leading to UFRJ50816 relative to the standard S. paradoxus karyotype that account for most of this reproductive isolation [13, 17, 44]. To test whether the recent proliferation of of Tsu4 in UFRJ50816 has induced genome rearrangements involved in reproductive isolation, I identified translocation breakpoints in S. paradoxus UFRJ50816 relative to the standard karyotype S. paradoxus strain CBS432 using Mummer [45] and Ribbon [46]. Only one out of five translocations showed clear evidence for Tsu4 sequences at both breakpoints. Intriguingly, both breakpoints of the translocation between chrIX and chrXV (which has recently been shown to reduce spore viability by approximately $50 \%$ [44]) each contained a full-length Tsu4 element (Fig. 1b). These two elements are from the major clade of Tsu4 sequences found only in UFRJ50816, and are oriented in the directions expected if they were involved in a reciprocal exchange event. These results indicate that recent ectopic exchange among Tsu4 sequences is not the primary cause of the majority of translocations in UFRJ50816, however Tsu4 proliferation may have facilitated some genome rearrangements in the UFRJ50816 lineage.

In conclusion, here I report evidence for a novel HTT event in fungi involving Tsu4 in S. paradoxus based on (i) unexpectedly high similarity between Tsu4 elements in S. paradoxus and S. uvarum, (ii) a patchy distribution of Tsu4 in S. paradoxus and general absence from its sister species $S$. cerevisiae, and (iii) discordance between the phylogenetic history of Tsu4 sequences and host species trees. Based on available data, the most parsimonious scenario for the evolution of the Ty4/Tsu4 super-family is that an ancestral Ty4/Tsu4-like element was present in the ancestor of the Saccharomyces sensu stricto group, which diversified into $T y 4$ along the lineage leading to S. cerevisiae/S. paradoxus and into Tsu4 on the lineage leading to $S$. uvarum/S. eubayanus. Subsequently, a HTT event occurred introducing Tsu4 into the ancestor of nonOld World S. paradoxus, most likely from S. uvarum, S. eubayanus or a related species. This scenario is plausible since both $S$. uvarum and S. eubayanus have been sampled from sites in N. America and S. America that overlap or are in close proximity to the predicted range of S. paradoxus [33, 35, 47-49], and S. uvarum has been isolated from the same field sites as $S$. paradoxus in N. America [50]. Other more complex scenarios are also possible but would involve additional HTT events, such as HTT from S. uvarum/S. eubayanus to S. paradoxus via intermediate species or multiple HTT events from an unidentified species into the ancestor of non-Old World $S$. paradoxus and S. uvarum, S. eubayanus or their common ancestor. A number of open questions remain about the Tsu4 HTT event, including which lineage donated Tsu4 to S. paradoxus, where the HTT event occurred, how widely Tsu4 has spread in S. paradoxus, and whether the HTT event was mediated by interspecific hybridization or some other mechanism. I also show that full-length Tsu4 elements are associated with the breakpoints of a reciprocal translocation that provides partial reproductive isolation 
between lineages of S. paradoxus from S. America and the rest of the world. These findings together with related work on Ty2 in S. cerevisiae $[8,11,51]$ provide support for the hypothesis that HTT and subsequent proliferation can induce genome rearrangements that contribute to post-zygotic isolation in yeast.

\section{Additional files}

Additional file 1: Ty content in S. cerevisiae and S. paradoxus PacBio assemblies. Numbers of full-length elements (both LTRs and internal region present with $>95 \%$ coverage of canonical internal region), truncated elements (internal region present with $<95 \%$ coverage of canonical internal region), or solo LTRs (no match to internal region) were identified using a RepeatMasker (version 4.0.5) based strategy and a custom library of Ty elements from [1 1] supplemented with S. paradoxus Tsu4. (TSV $1.20 \mathrm{~kb}$ )

Additional file 2: Coordinates of Ty elements in S. cerevisiae and S. paradoxus PacBio assemblies. Zip file of BED-formatted genome annotations for 12 strains of S. cerevisiae and S. paradoxus with PacBio assemblies. Full-length elements (f), truncated elements (t), or solo LTRs ( $\mathrm{s}$ ) were identified using a RepeatMasker (version 4.0.5) based strategy and a custom library of Ty elements from [11] supplemented with S. paradoxus Tsu4. (ZIP $91.1 \mathrm{~kb}$ )

Additional file 3: Summary of complete or nearly-complete Tsu4 sequences identified in Saccharomyces whole genome assemblies at NCBI. Accession number and coordinates of 12 complete or nearly-complete Tsu4 elements identified by BLAST in 392 Saccharomyces (taxid: 4930) WGS assemblies at NCBI that had high similarity ( $>80 \%$ coverage and $>80 \%$ identity) to the Tsu4 query sequence (Genbank: AJ439550). (CSV $1.77 \mathrm{~kb}$ )

Additional file 4: Multiple sequence alignment of Ty4 and Tsu4 elements in Saccharomyces sensu stricto species. Multiple sequence alignment of all full-length Ty4/Tsu4 elements from 12 strains of S. cerevisiae and S. paradoxus with PacBio assemblies from Yue et al. [13] plus all complete or nearly-complete Tsu4 elements identified in Saccharomyces WGS assemblies at $\mathrm{NCBI}$ (> $>80 \%$ coverage and $>80 \%$ identity relative to the Tsu4 query sequence from S. uvarum). Fasta files of Ty4/Tsu4 sequences from all strains plus the Ty4 and Tsu4 query sequences were concatenated together and aligned using MAFFT (version 7.273-e; options: -thread 28) [52]. (TXT $335 \mathrm{~kb})$

Additional file 5: Maximum likelihood tree file for Ty4 and Tsu4 elements in Saccharomyces sensu stricto species. Newick-formatted file of the maximum-likelihood tree of all full-length Ty4/Tsu4 elements from 16 strains of S. cerevisiae and S. paradoxus plus all complete or nearly-complete Tsu4 elements identified in Saccharomyces WGS assemblies at NCBI. Maximum-likelihood phylogenetic analysis was performed on the multiple alignment in Additional file 4 using RAxML (version: 8.2.4; options - T 28 - $\mathrm{f} \mathrm{a}$ -x 12345 -p 12345 -N 100 -m GTRGAMMA) [28] excluding positions 1-166 and 6086-6476. (TXT 3.79 kb)

Additional file 6: Coordinates of best-matches to scaffolds from $S$. pastorianus and Saccharomyces sp. M14 containing Tsu4 sequences vs. a pan-genome of S. eubayanus and S. cerevisiae genomes. S. pastorianus and Saccharomyces sp. M14 scaffolds were aligned to a pan-genome composed of scaffolds from S. eubayanus FM1318 (Genbank: GCF_001298625.1) and chromosomes from S. cerevisiae S288c (from [13]). Alignments were generated using nucmer (default parameters), delta-filter (options: -1 -1 2000), and show-coords (options: -ITH) in mummer 3.23 [45]. (TSV 5.15 kb)

Additional file 7: Dot-plot of the Chinese lager strain Saccharomyces sp. M14 vs. a pan-genome of S. eubayanus and S. cerevisiae genomes. Dot-plot of Saccharomyces sp. M14 scaffolds aligned to a pan-genome composed of scaffolds from S. eubayanus FM1318 (Genbank: GCF_001298625.1) and chromosomes from S. cerevisiae S288c (from [13]), showing that Saccharomyces sp. M14 contains subgenomes from both species and that this strain may be a previously-unidentified strain of the lager brewing species S. pastorianus. The dot-plot was generated using nucmer (default parameters) and mummerplot (options: --size large -fat --color - $f$--png) in mummer 3.23 [45]. (PDF $58.8 \mathrm{~kb}$ )

Additional file 8: Dot-plot of the S. pastorianus group 2/Frohberg strain W34/70 vs. a pan-genome of $S$. eubayanus and S. cerevisiae genomes. Dot-plot of S. pastorianus group 2/Frohberg strain W34/70 scaffolds aligned to a pan-genome composed of scaffolds from S. eubayanus (Genbank: GCF_001298625.1) and chromosomes from S. cerevisiae (S288C from [13]), showing that S. pastorianus group 2/Frohberg strain W34/70 contains subgenomes from both species in a similar pattern as for Saccharomyces sp. M14 (see Additional file 7). The dot-plot was generated using nucmer (default parameters) and mummerplot (options: --size large -fat --color -f --png) in mummer 3.23 [45]. (PDF 69.8 kb)

\section{Abbreviations}

HTT: Horizontal TE transfer; LTR: Long terminal repeat; TE: Transposable element; TSD: Target site duplication

\section{Acknowledgements}

CMB thanks Shan-ho Tsai and Yecheng Huang for bioinformatics application support; the Georgia Advanced Computing Resource Center for computing time; and Guilherme Dias, Douda Bensasson, and two anonymous reviewers for comments on the manuscript.

\section{Funding}

CMB was supported by the University of Georgia Research Foundation.

\section{Availability of data and materials}

Canonical LTR and annotated internal sequences for S. paradoxus Tsu4 have been submitted to Repbase. All other data generated or analyzed during this study are included in this published article and its supplementary information files.

\section{Author's contributions}

$\mathrm{CMB}$ conceived of the project, performed the research, analyzed the data, and wrote the paper. The author read and approved the final manuscript.

\section{Ethics approval and consent to participate}

Not applicable.

\section{Competing interests}

The author declares that he has no competing interests.

\section{Publisher's Note}

Springer Nature remains neutral with regard to jurisdictional claims in published maps and institutional affiliations.

Received: 2 April 2018 Accepted: 31 May 2018

Published online: 12 June 2018

\section{References}

1. Schaack S, Gilbert C, Feschotte C. Promiscuous DNA: horizontal transfer of transposable elements and why it matters for eukaryotic evolution. Trends Ecol Evol (Amst). 2010;25(9):537-46.

2. Wallau GL, Ortiz MF, Loreto ELS. Horizontal transposon transfer in eukarya: detection, bias, and perspectives. Genome Biol Evol. 2012;4(8):801-11.

3. Wallau GL, Vieira C, Loreto ELS. Genetic exchange in eukaryotes through horizontal transfer: connected by the mobilome. Mob DNA. 2018;9:6.

4. Daniels SB, Peterson KR, Strausbaugh LD, Kidwell MG, Chovnick A. Evidence for horizontal transmission of the $\mathrm{P}$ transposable element between Drosophila species. Genetics. 1990;124(2):339-55.

5. Dotto BR, Carvalho EL, Silva AF, Silva D, Fernando L, Pinto PM, Ortiz MF, Wallau GL. HTT-DB: Horizontally transferred transposable elements database. Bioinformatics. 2015;31(17):2915-7.

6. Dobinson KF, Harris RE, Hamer JE. Grasshopper, a long terminal repeat (LTR) retroelement in the phytopathogenic fungus Magnaporthe grisea. Mol Plant Microbe Interact. 1993;6(1):114-26.

7. Daboussi M-J, Davière J-M, Graziani S, Langin T. Evolution of the Fot1 transposons in the genus Fusarium: discontinuous distribution and epigenetic inactivation. Mol Biol Evol. 2002;19(4):510-20. 
8. Liti G, Peruffo A, James SA, Roberts IN, Louis EJ. Inferences of evolutionary relationships from a population survey of LTR-retrotransposons and telomeric-associated sequences in the Saccharomyces sensu stricto complex. Yeast. 2005;22(3):177-92.

9. Novikova O, Fet $\mathrm{V}$, Blinov A. Non-LTR retrotransposons in fungi. Funct Integr Genomics. 2009;9(1):27-42.

10. Amyotte SG, Tan X, Pennerman K, del Mar Jimenez-Gasco M, Klosterman SJ, Ma L-J, Dobinson KF, Veronese P. Transposable elements in phytopathogenic Verticillium spp.: insights into genome evolution and inter- and intra-specific diversification. BMC Genomics. 2012;13:314.

11. Carr M, Bensasson D, Bergman CM. Evolutionary genomics of transposable elements in Saccharomyces cerevisiae. PLoS ONE. 2012;7(11):50978

12. Sarilar V, Bleykasten-Grosshans C, Neuveglise C. Evolutionary dynamics of hAT DNA transposon families in Saccharomycetaceae. Genome Biol Evol. 2015;7(1):172-90.

13. Yue J-X, Li J, Aigrain L, Hallin J, Persson K, Oliver K, Bergstrom A, Coupland P, Warringer J, Lagomarsino MC, Fischer G, Durbin R, Liti G. Contrasting evolutionary genome dynamics between domesticated and wild yeasts. Nat Genet. 2017;49(6):913-24.

14. Stucka R, Lochmäller H, Feldmann H. Ty4, a novel low-copy number element in Saccharomyces cerevisiae: one copy is located in a cluster of Ty elements and tRNA genes. Nucleic Acids Res. 1989;17(13):4993-5002.

15. Kim JM, Vanguri S, Boeke J, Gabriel A, Voytas DF. Transposable elements and genome organization: a comprehensive survey of retrotransposons revealed by the complete Saccharomyces cerevisiae genome sequence. Genome Res. 1998;8(5):464-78.

16. Bleykasten-Grosshans C, Friedrich A, Schacherer J. Genome-wide analysis of intraspecific transposon diversity in yeast. BMC Genomics. 2013;14:399.

17. Fischer G, James SA, Roberts IN, Oliver SG, Louis EJ. Chromosomal evolution in Saccharomyces. Nature. 2000;405(6785):451-4.

18. Neuveglise C, Feldmann H, Bon E, Gaillardin C, Casaregola S. Genomic evolution of the long terminal repeat retrotransposons in hemiascomycetous yeasts. Genome Res. 2002;12(6):930-43.

19. Ellinghaus $D$, Kurtz S, Willhoeft U. LTRharvest, an efficient and flexible software for de novo detection of LTR retrotransposons. BMC Bioinformatics. 2008;9(1):18.

20. Loytynoja A, Goldman N. Phylogeny-aware gap placement prevents errors in sequence alignment and evolutionary analysis. Science. 2008:320(5883):1632-5.

21. Yang Z. PAML 4: Phylogenetic Analysis by Maximum Likelihood. Mol Biol Evol. 2007;24(8):1586-91.

22. Huerta-Cepas J, Serra F, Bork P. ETE 3: Reconstruction, analysis, and visualization of phylogenomic data. Mol Biol Evol. 2016;33(6):1635-8.

23. Scannell DR, Zill OA, Rokas A, Payen C, Dunham MJ, Eisen MB, Rine J, Johnston M, Hittinger CT. The awesome power of yeast evolutionary genetics: New genome sequences and strain resources for the Saccharomyces sensu stricto genus. G3. 2011;1(1):11-25.

24. Fink G, Boeke J, Garfinkel D. The mechanism and consequences of retrotransposition. Trends Genet. 1986;2:118-23.

25. Liti G, Carter DM, Moses AM, Warringer J, Parts L, James SA, Davey RP, Roberts IN, Burt A, Koufopanou V, Tsai IJ, Bergman CM, Bensasson D, O'Kelly MJT, Oudenaarden Av, Barton DBH, Bailes E, Nguyen AN, Jones M, Quail MA, Goodhead I, Sims S, Smith F, Blomberg A, Durbin R, Louis EJ. Population genomics of domestic and wild yeasts. Nature. 2009;458(7236):337-41.

26. Nelson MG, Linheiro RS, Bergman CM. McClintock: an integrated pipeline for detecting transposable element insertions in whole-genome shotgun sequencing data. G3. 2017;7:2749-62.

27. Marsit S, Mena A, Bigey F, Sauvage F-X, Couloux A, Guy J, Legras J-L, Barrio E, Dequin S, Galeote V. Evolutionary advantage conferred by an eukaryote-to-eukaryote gene transfer event in wine yeasts. Mol Biol Evol. 2015;32(7):1695-707.

28. Stamatakis A. RAxML version 8: a tool for phylogenetic analysis and post-analysis of large phylogenies. Bioinformatics. 2014;30(9):1312-3.

29. Cliften P, Sudarsanam P, Desikan A, Fulton L, Fulton B, Majors J, Waterston R, Cohen BA, Johnston M. Finding functional features in Saccharomyces genomes by phylogenetic footprinting. Science. 2003;301(5629):71-6.

30. Okuno M, Kajitani R, Ryusui R, Morimoto H, Kodama $Y$, Itoh T. Next-generation sequencing analysis of lager brewing yeast strains reveals the evolutionary history of interspecies hybridization. DNA Res. 2016;23(1):67-80

31. Liu C, Li Q, Niu C, Zheng F, Li Y, Zhao Y, Yin X. Genome sequence of the lager-brewing yeast Saccharomyces sp. strain M14, used in the high-gravity brewing industry in China. Genome Announc. 2017;5(43): e01194-17.

32. Borneman AR, Pretorius IS. Genomic insights into the Saccharomyces sensu stricto complex. Genetics. 2015;199(2):281-91.

33. Libkind $D$, Hittinger $C T$, Valerio $E$, Goncalves $C$, Dover J, Johnston $M$, Goncalves P, Sampaio JP. Microbe domestication and the identification of the wild genetic stock of lager-brewing yeast. Proc Natl Acad Sci USA. 2011;108(35):14539-44.

34. Baker E, Wang B, Bellora N, Peris D, Hulfachor AB, Koshalek JA, Adams $M$, Libkind $D$, Hittinger $C T$. The genome sequence of Saccharomyces eubayanus and the domestication of lager-brewing yeasts. Mol Biol Evol. 2015;32(11):2818-31.

35. Peris D, Langdon QK, Moriarty RV, Sylvester K, Bontrager M, Charron G, Leducq J-B, Landry CR, Libkind D, Hittinger CT. Complex ancestries of lager-brewing hybrids were shaped by standing variation in the wild yeast Saccharomyces eubayanus. PLOS Genet. 2016;12(7):1006155.

36. Liti $\mathrm{G}$, Barton $\mathrm{DBH}$, Louis EJ. Sequence diversity, reproductive isolation and species concepts in Saccharomyces. Genetics. 2006;174(2):839-50.

37. Doniger SW, Kim HS, Swain D, Corcuera D, Williams M, Yang S-P, Fay JC. A catalog of neutral and deleterious polymorphism in yeast. PLOS Genet. 2008;4(8):1000183.

38. Muller LAH, McCusker JH. A multi-species based taxonomic microarray reveals interspecies hybridization and introgression in Saccharomyces cerevisiae. FEMS Yeast Res. 2009;9(1):143-52.

39. Strope PK, Skelly DA, Kozmin SG, Mahadevan G, Stone EA, Magwene PM, Dietrich FS, McCusker JH. The 100-genomes strains, an S, cerevisiae resource that illuminates its natural phenotypic and genotypic variation and emergence as an opportunistic pathogen. Genome Res. 2015;25(5): $762-74$.

40. Almeida P, Barbosa R, Bensasson D, Goncalves P, Sampaio JP. Adaptive divergence in wine yeasts and their wild relatives suggests a prominent role for introgressions and rapid evolution at noncoding sites. Mol Ecol. 2017;26(7):2167-82

41. Peter J, Chiara MD, Friedrich A, Yue J-X, Pflieger D, Bergström A, Sigwalt A, Barre B, Freel K, Llored A, Cruaud C, Labadie K, Aury J-M, Istace B, Lebrigand K, Barbry P, Engelen $\mathrm{S}$, Lemainque A, Wincker $\mathrm{P}$, Liti G, Schacherer J. Genome evolution across 1011 Saccharomyces cerevisiae isolates. Nature. 2018;556:339-44.

42. Naumov GI, Naumova ES, Hagler AN, Mendonca-Hagler LC, Louis EJ. A new genetically isolated population of the Saccharomyces sensu stricto complex from Brazil. Antonie van Leeuwenhoek. 1995;67(4):351-5.

43. Naumov GI, James SA, Naumova ES, Louis EJ, Roberts IN. Three new species in the Saccharomyces sensu stricto complex: Saccharomyces cariocanus, Saccharomyces kudriavzevii and Saccharomyces mikatae. Int Syst Evol Microbiol. 2000;50 Pt 5:1931-42.

44. Almutawa Q. Impact of Chromosomal Translocations (CTs) on reproductive isolation and fitness in natural yeast isolates. PhD thesis, University of Manchester. 2016. https://www.escholar.manchester.ac.uk/ item/?pid=uk-ac-man-scw:307192. Accessed 7 June 2018.

45. Kurtz S, Phillippy A, Delcher AL, Smoot M, Shumway M, Antonescu C, Salzberg SL. Versatile and open software for comparing large genomes. Genome Biol. 2004;5(2):12.

46. Nattestad M, Chin C-S, Schatz MC. Ribbon: Visualizing complex genome alignments and structural variation. bioRxiv. 2016;082123. https://doi.org/ 10.1101/082123. Accessed 7 June 2018.

47. Almeida P, Goncalves C, Teixeira S, Libkind D, Bontrager M, Masneuf-Pomarede I, Albertin W, Durrens P, Sherman DJ, Marullo P, Todd Hittinger C, Goncalves P, Sampaio JP. A Gondwanan imprint on global diversity and domestication of wine and cider yeast Saccharomyces uvarum. Nat Commun. 2014;5:4044.

48. Peris D, Sylvester K, Libkind D, Gonçalves P, Sampaio JP, Alexander WG, Hittinger CT. Population structure and reticulate evolution of Saccharomyces eubayanus and its lager-brewing hybrids. Mol Ecol. 2014:23(8):2031-45.

49. Robinson HA, Pinharanda A, Bensasson D. Summer temperature can predict the distribution of wild yeast populations. Ecol Evol. 2016;6(4): $1236-50$. 
50. Sampaio JP, Goncalves P. Natural populations of Saccharomyces kudriavzevii in Portugal are associated with oak bark and are sympatric with S, cerevisiae and S. paradoxus. Appl Environ Microbiol. 2008;74(7): 2144-52.

51. Hou J, Friedrich A, de Montigny J, Schacherer J. Chromosomal rearrangements as a major mechanism in the onset of reproductive isolation in Saccharomyces cerevisiae. Curr Biol. 2014;24(10):1153-9.

52. Katoh $\mathrm{K}$, Kuma K, Toh $\mathrm{H}$, Miyata T. MAFFT version 5: improvement in accuracy of multiple sequence alignment. Nucleic Acids Res. 2005;33(2): $511-8$.

- fast, convenient online submission

- thorough peer review by experienced researchers in your field

- rapid publication on acceptance

- support for research data, including large and complex data types

- gold Open Access which fosters wider collaboration and increased citations

- maximum visibility for your research: over $100 \mathrm{M}$ website views per year

At $B M C$, research is always in progress.

Learn more biomedcentral.com/submissions 